\title{
Nanodroplets of Polymer Solutions on Solid Surfaces: Equilibrium Structures and Solvent Evaporation
}

\author{
Vitaly S. Kravchenko ${ }^{\mathrm{a}, \mathrm{b}, \mathrm{c}}$ and Igor I. Potemkin ${ }^{\mathrm{a}, \mathrm{b}, \mathrm{d}, *}$
}

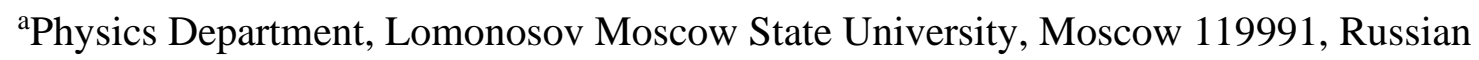
Federation

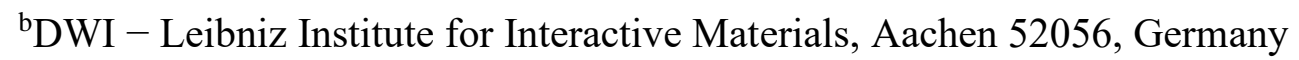

cA. N. Nesmeyanov Institute of Organoelement Compounds, Russian Academy of Sciences, Moscow 119991, Russian Federation

${ }^{\mathrm{d} N a t i o n a l ~ R e s e a r c h ~ S o u t h ~ U r a l ~ S t a t e ~ U n i v e r s i t y, ~ C h e l y a b i n s k ~ 454080, ~ R u s s i a n ~ F e d e r a t i o n ~}$

*Corresponding author, e-mail: igor@polly.phys.msu.ru (I.I.P.) 


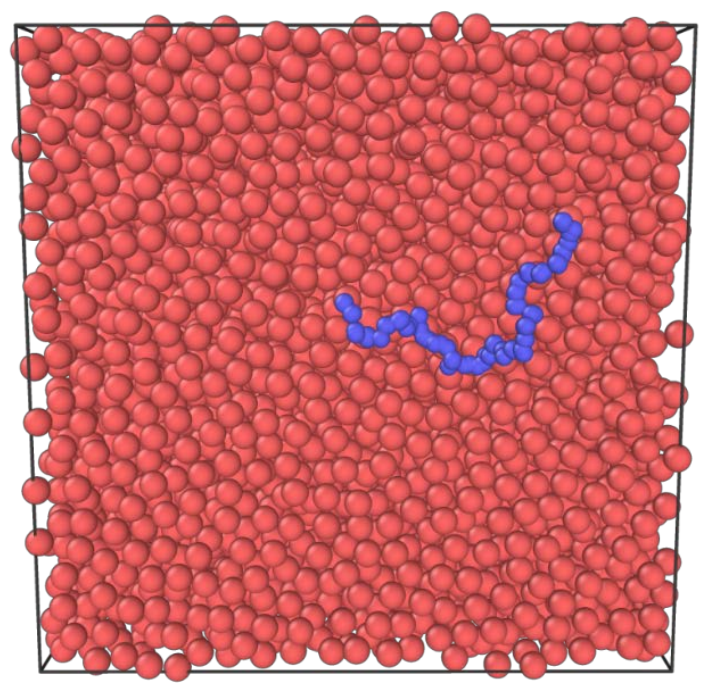

Figure S1. Snapshot of the single polymer chain in solution having the same density as in the droplet.

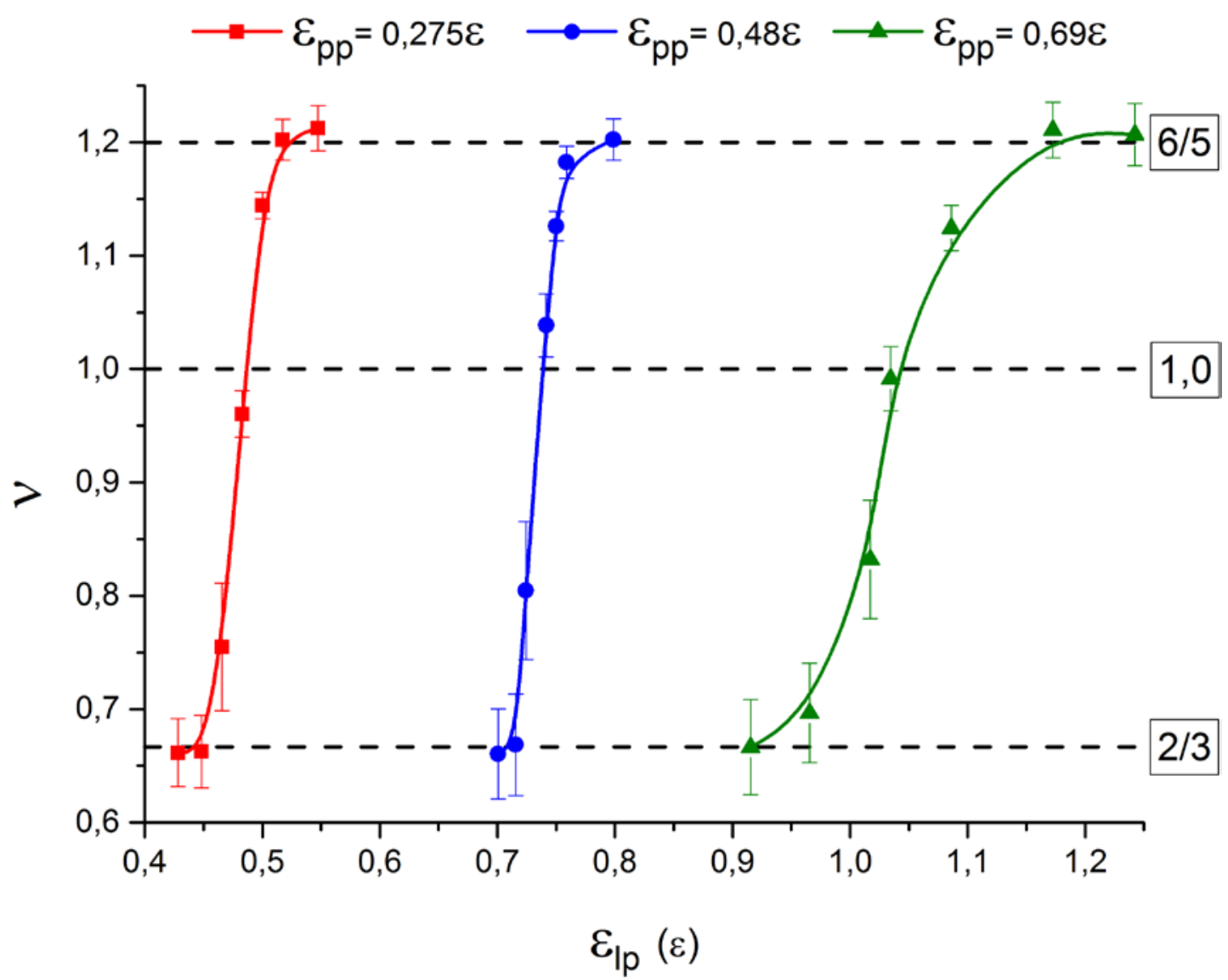

Figure S2. Power $v,\left\langle R^{2}\right\rangle \sim N^{v}$, as a function of the liquid-polymer interaction parameter $\varepsilon_{l p}$ at different values of the polymer-polymer interaction parameter $\varepsilon_{p p}$. 


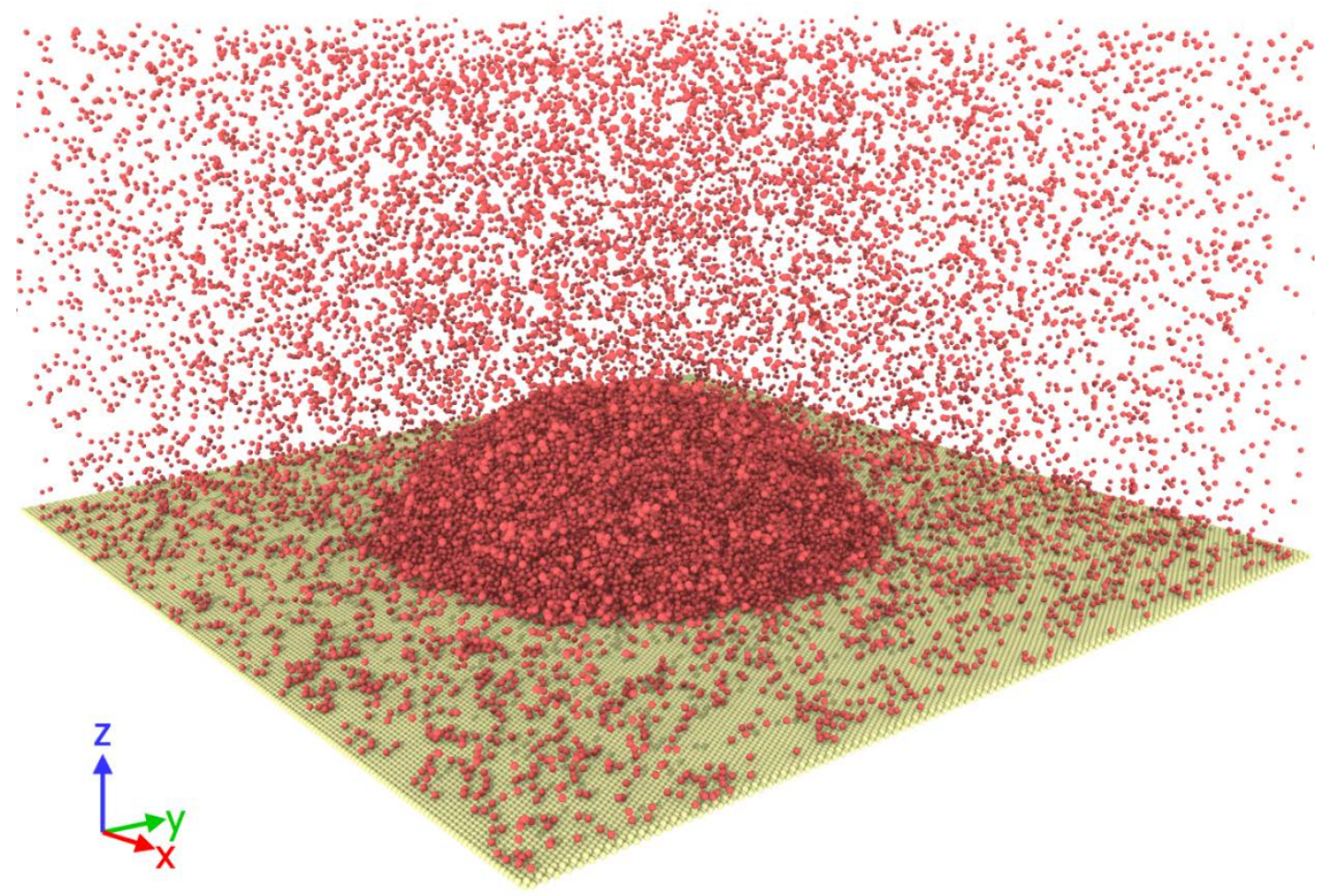

Figure S3. Snapshot of a solvent droplet formed by $10^{5}$ beads placed on the surface made of the LennardJones beads.

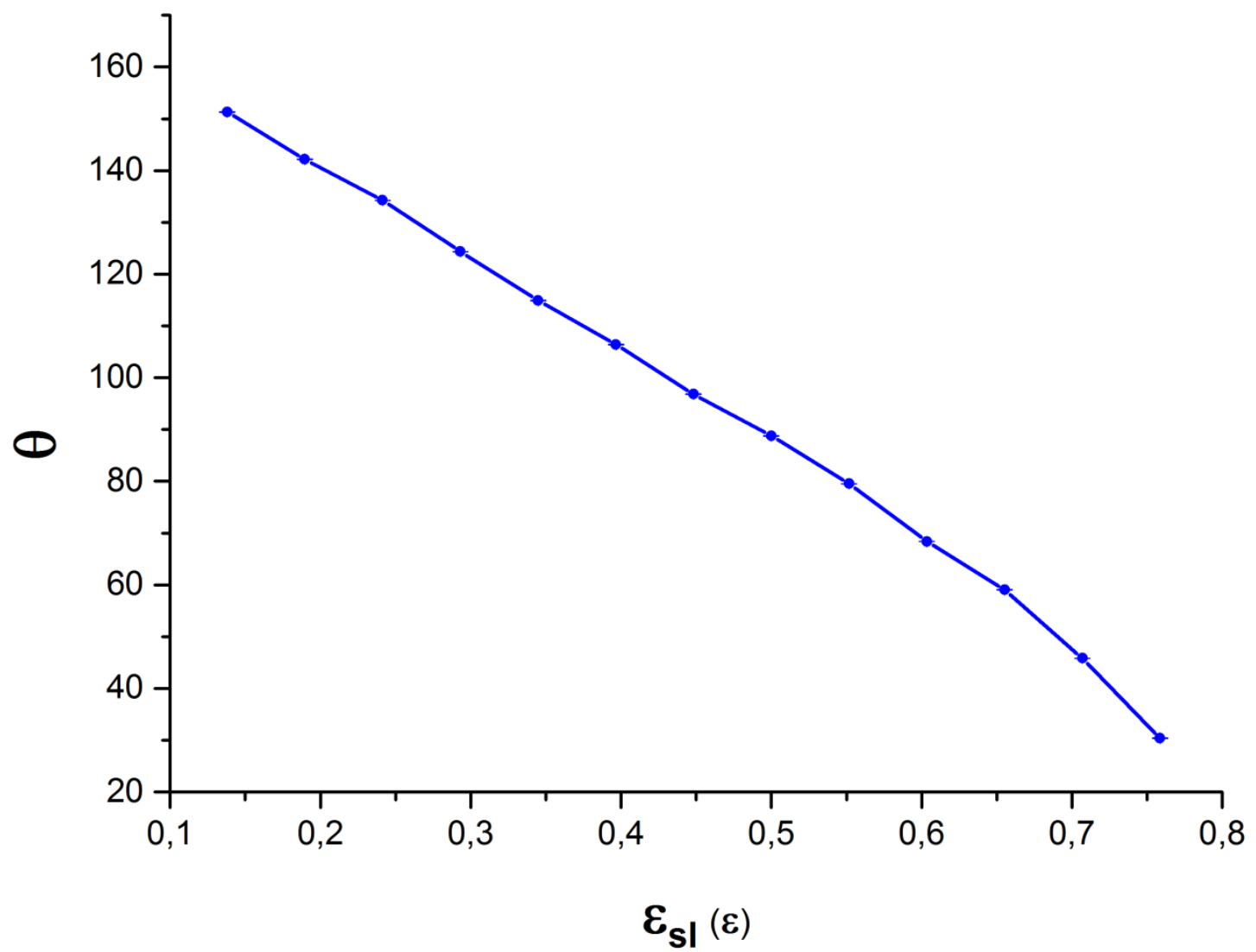

Figure S4. The contact angle $\theta$ as a function of the surface-liquid interaction parameter $\varepsilon_{s l}$ for the system with the surface made of the Lennard-Jones beads. 


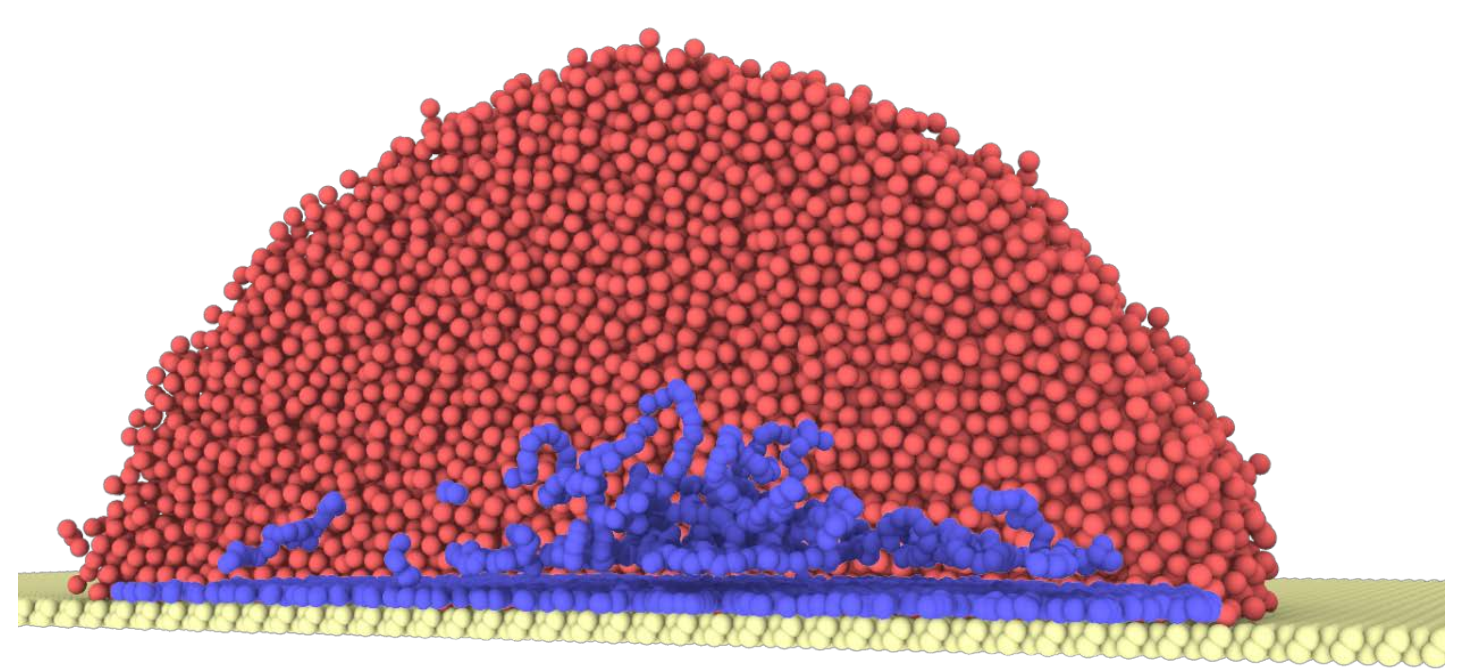

Figure S5. Snapshot (side view) of the droplet with polymer chains of the length $l=55 \sigma$ at $\varphi=10 \%$, $\varepsilon_{s l}=0,4 \varepsilon, \varepsilon_{s p}=1,05 \varepsilon, \varepsilon_{l p}=1,17 \varepsilon$ and $\varepsilon_{p p}=0,69 \varepsilon$ for the system with the surface made of the Lennard-Jones beads. There are $5 \times 10^{4}$ beads in the system.

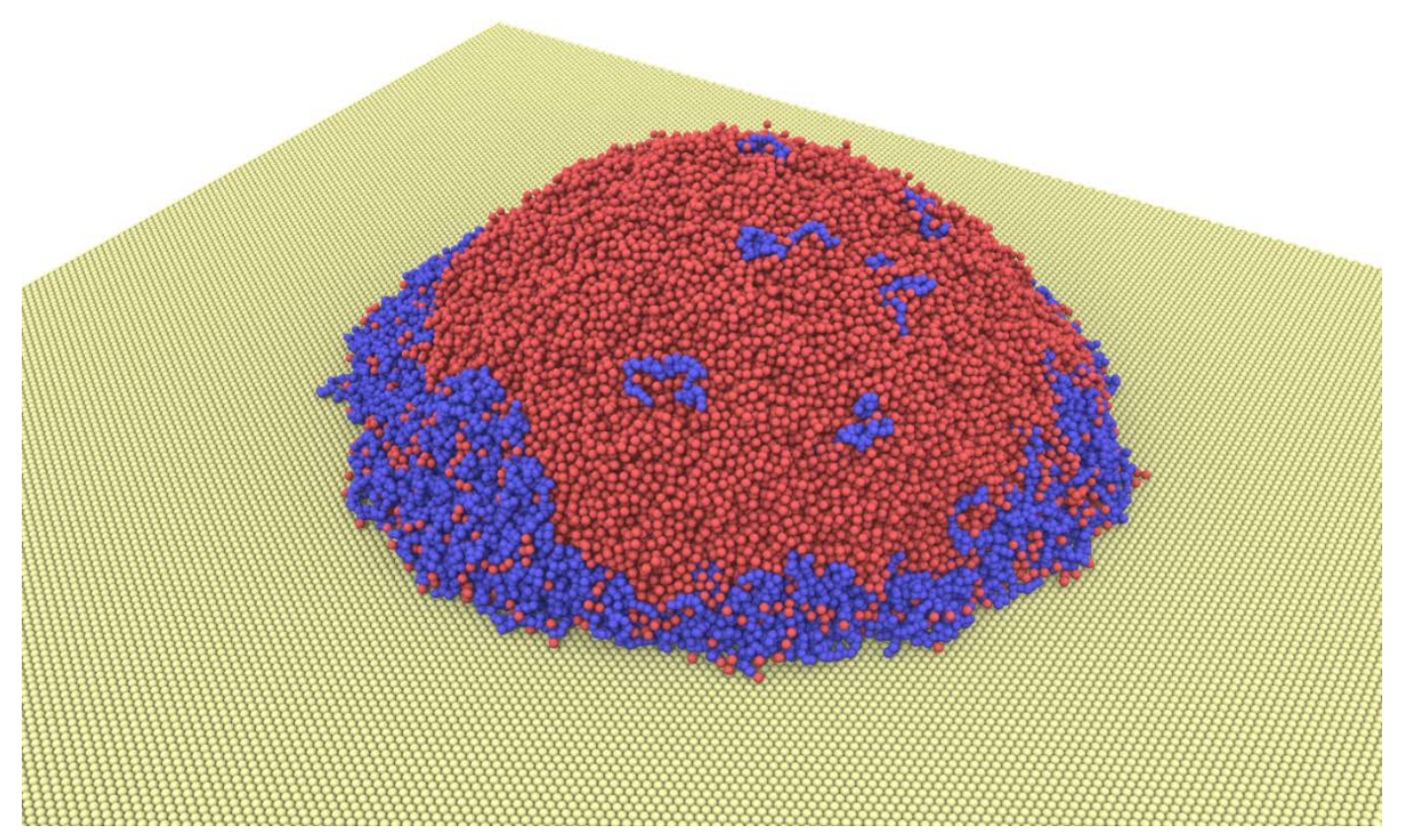

Figure S6. Snapshot of the droplet with the polymer chains near the contact line at $=55 \sigma, \varphi=15 \%$, $\varepsilon_{s l}=0,6 \varepsilon, \varepsilon_{s p}=0,4 \varepsilon, \varepsilon_{l p}=0,485 \varepsilon$ and $\varepsilon_{p p}=0,28 \varepsilon$ for the system with the surface made of the Lennard-Jones beads. There are $10^{5}$ beads in the system. 

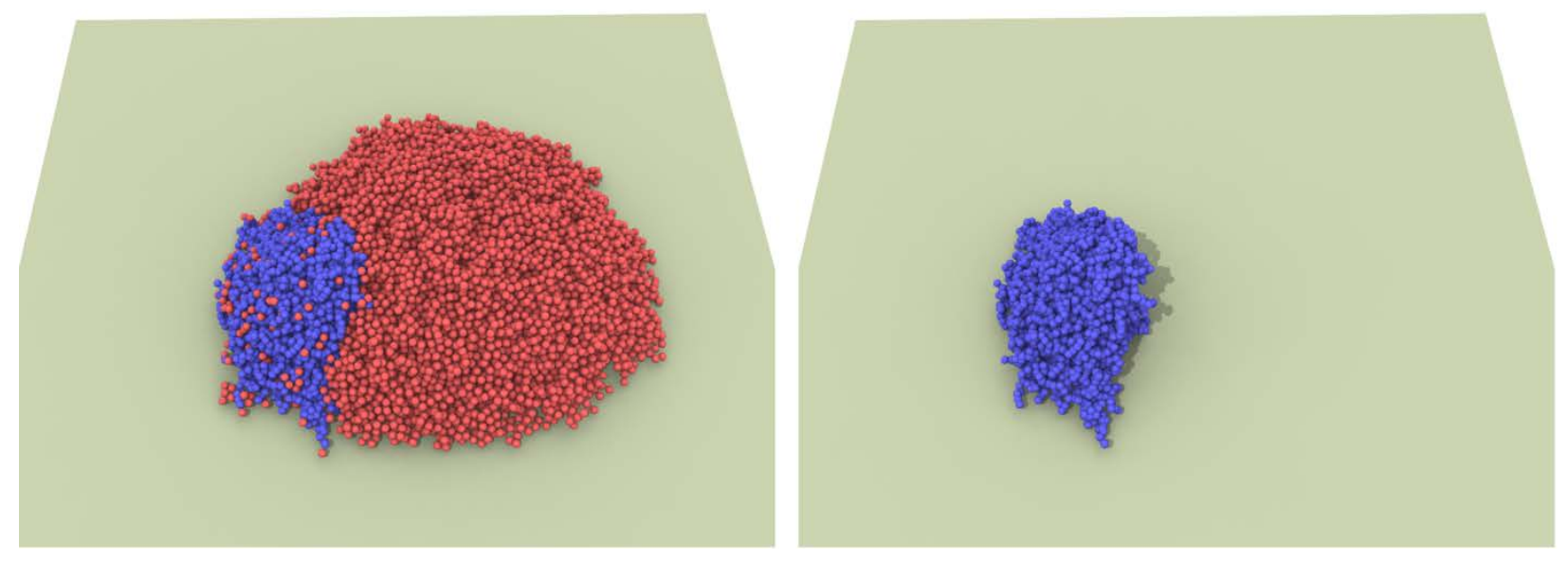

Figure S7. Snapshots of the droplet with the polymer: liquid' beads are visible (left) and not (right). $l=$ $55 \sigma, \varphi=20 \%, \varepsilon_{s l}=3,1 \varepsilon, \varepsilon_{s p}=1,6 \varepsilon, \varepsilon_{l p}=0,44 \varepsilon$ and $\varepsilon_{p p}=0,3 \varepsilon$. There are $2 \times 10^{4}$ beads in the system.
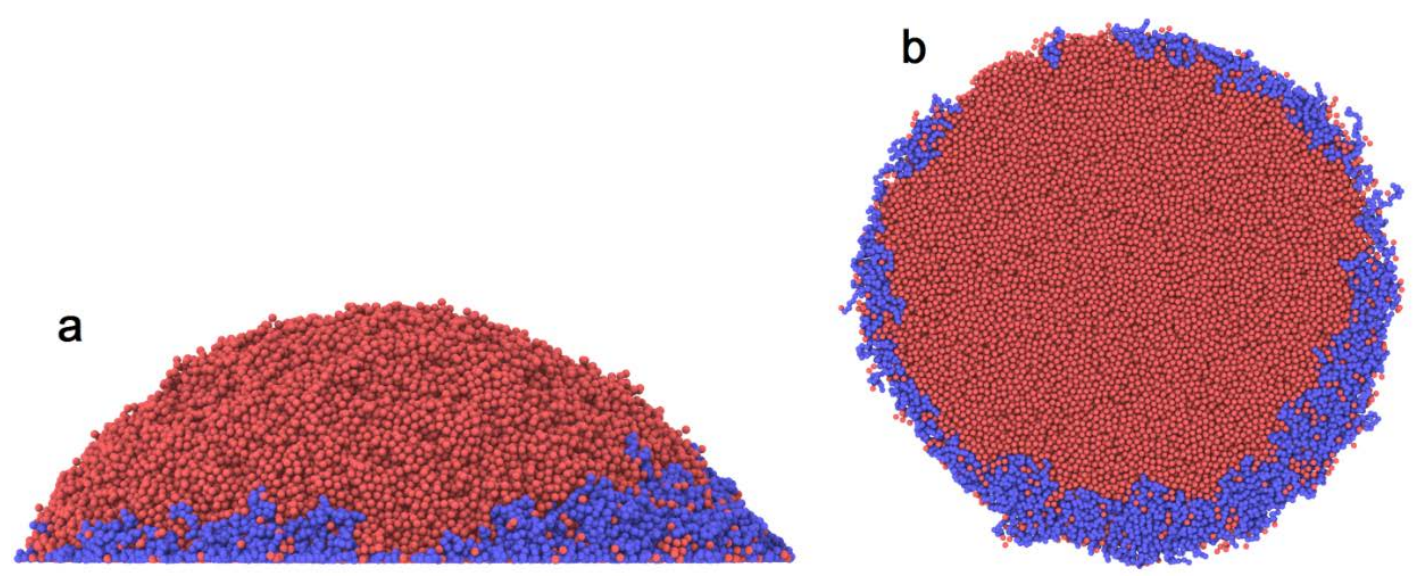

Figure S8. Snapshots of the droplet with the polymer chains near the contact line: side view (a) and bottom view (b). $l=55 \sigma, \varphi=10 \%, \varepsilon_{s l}=2,75 \varepsilon, \varepsilon_{s p}=1,6 \varepsilon, \varepsilon_{l p}=0,46 \varepsilon$ and $\varepsilon_{p p}=0,28 \varepsilon$. There are $10^{5}$ beads in the system. 


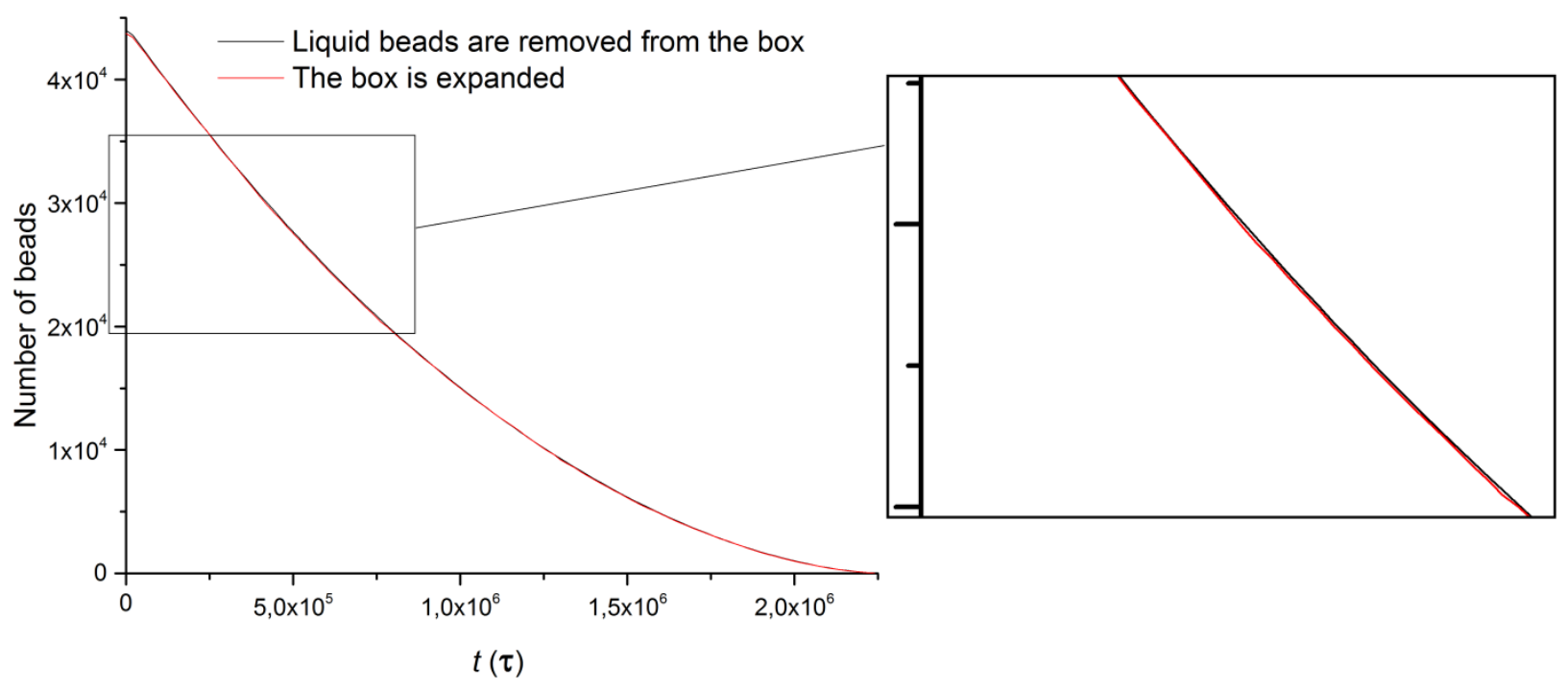

Figure S9. Time evolution of the number of beads inside the droplet for two different methods of evaporation. There are $5 \times 10^{4}$ beads in the system and $\varepsilon_{s l}=1,7 \varepsilon$. 